\title{
Predictors of Blood Culture Positivity in Pediatric Brucellosis
}

\author{
Soner Sertan Kara ${ }^{1}$ and Yasemin Cayir 2
}

\begin{abstract}
Objective: To investigate the predictors of blood culture positivity in children with brucellosis.

Study Design: Descriptive study.

Place and Duration of Study: Department of Pediatric Infectious Diseases in Erzurum Regional Training and Research Hospital, Turkey, from January to December 2015.

Methodology: Eighty-six children under 16 years of age, with brucellosis, were retrospectively evaluated. Compatible clinical findings plus presence of positivity at titers of $\geq 1: 160$ in serum and/or Coombs agglutination tests in a single serum sample and/or a minimum fourfold increase within a 2-3 week interval were diagnostic for brucellosis. Only patients with blood cultures were included. Patients' demographical, clinical, and laboratory risk factors, such as age, gender, presence and duration of symptoms, and laboratory characteristics were analysed.

Results: Brucella spp. grew in blood cultures of $24(27.9 \%)$ patients. Children with blood culture positivity had shorter symptom duration than those with negative blood cultures $(p=0.03)$. Absence of personal and household histories of brucellosis $(p=0.02$ and $p=0.04$, respectively), lower hemoglobin, iron, and vitamin $D(p<0.001, p=0.006$, and $p=0.006$, respectively), and higher leukocyte, CRP, and ferritin $(p<0.001, p=0.001$, and $p<0.001$, respectively) levels were associated with isolation of Brucella spp. in blood culture. Children with positive blood cultures had higher serum tube and Coombs agglutination test results $(p=0.001$ and $p<0.001)$. ROC analysis showed that ferritin at a cut-off level of $122 \mathrm{ng} / \mathrm{mL}$ (Cl 95\% 0.86-0.97, $\mathrm{p}<0.001)$ and Brucella Coombs agglutination test at a cut-off level of $1 / 480(\mathrm{Cl} 95 \% 0.84-0.96$, $\mathrm{p}<0.001$ ) were the most sensitive and specific predictors of bacteremia.

Conclusion: Serum hemoglobin, iron, ferritin, vitamin D, and C-reactive protein levels, Brucellar tube and Coombs agglutination tests, and leukocyte count could help to predict definitive diagnosis in pediatric brucellosis when molecular techniques are not feasible, such as in source-limited countries.
\end{abstract}

Key Words: Blood culture, Brucellosis, Coombs agglutination test, Ferritin, Children.

\section{INTRODUCTION}

Infectious diseases follow a dynamic course over time. Various infections such as plague, variola, and poliomyelitis, which were once particularly predominant, have lost their importance, while others, such as human immunodeficiency virus, Ebola virus, and Zika virus have emerged during recent decades. Brucellosis is a striking example of term-independent disease. It is the most widespread bacterial zoonotic disease and is caused by Brucella species, with an annual estimated prevalence of more than 0.5 million new cases world-wide. ${ }^{1}$ The disease can have disabling conse-quences, and remains a significant economic and public health problem, particularly in Mediterranean countries, including Turkey.

Diagnosis of brucellosis relies on clinical presentations and laboratory tests. Serological methods precipitating

\footnotetext{
1 Department of Pediatric Infectious Diseases, Erzurum Regional Training and Research Hospital, Erzurum, Turkey

2 Department of Family Medicine, Ataturk University Medical Faculty, Erzurum, Turkey

Correspondence: Dr. Soner Sertan Kara, Department of

Pediatric Infectious Diseases, Erzurum Regional Training and Research Hospital, Erzurum, Turkey

E-mail:drsoner@yahoo.com

Received: July 24, 2018; Revised: November 26, 2018;

Accepted: December 13, 2018
}

presumptive diagnosis based on high or rising titers of Brucella-specific antibodies in serum are the most widely used diagnostic techniques. Although advantageous for being inexpensive and providing rapid test results, they possess risk of false positivity and negativity because of cross-reactivity with other Gram negative organisms, discordance with the clinical situation, inability to detect Brucella canis infections, and presence of blocking antibodies, or the prozone effect.2,3 Also, interpretation of the results can be challenging in endemic regions. Polymerase chain reaction (PCR) is a non-standardised and expensive method, which is not practical for developing countries. 4

Definitive diagnosis through isolation of Brucella species in cultures is well-known. It does not require expensive technology, highly equipped laboratories, or technical expertise, which are frequently unavailable in developing countries endemic for brucellosis. ${ }^{3}$ Nevertheless, delay in diagnosis is a constraint. Defining the risk factors related to blood culture positivity would help to go straight to the definitive diagnosis without losing time. In this study, the purpose was to identify the predictors affecting positive blood culture results in children with brucellosis in our source-limited country.

\section{METHODOLOGY}

Case files of 86 children with brucellosis, who presented to our tertiary care hospital in Erzurum, Turkey, during 
the time period from January to December 2015, were retrospectively evaluated. The included children were under 16 years of age, with the diagnosis of acute/ subacute/chronic brucellosis. Diagnosis of brucellosis was made on the basis of compatible clinical findings plus presence of positivity at titers of 1:160 and above in serum agglutination test (SAT) and/or Coombs agglutination test in a single serum sample and/or a minimum fourfold increase within a 2-3 week interval. ${ }^{5}$ Only patients with blood cultures were included. BACTEC 9120 (Becton Dickinson, Sparks, MD, USA) was used for cultures. If no growth was observed on the

Table I: Properties of 86 children with brucellosis.

\begin{tabular}{|c|c|}
\hline Property & $\mathrm{n}(\%)$ \\
\hline \multicolumn{2}{|l|}{ Demographic properties } \\
\hline Gender: Male / Female & $61(70.9 \%) / 25(29.1 \%)$ \\
\hline Duration of symptoms, days, median (IQR) & $30(15.7-60)$ \\
\hline Infection: Acute / Non-acute* & $67(77.9 \%) / 19(22.1 \%)$ \\
\hline Presence of previous brucellosis disease (relapse/reinfection) & $11(12.8 \%)$ \\
\hline Anti-brucellar treatment during the last month & $11(12.8 \%)$ \\
\hline Living place: Rural / Urban & $69(80.2 \%) / 17(19.8 \%)$ \\
\hline Consumption of raw milk and milk products & $76(88.4 \%)$ \\
\hline Animal husbandry & $69(80.2 \%)$ \\
\hline History of brucellosis in family members & $42(48.8 \%)$ \\
\hline \multicolumn{2}{|l|}{ Clinical features and physical examination findings } \\
\hline Arthralgia & $71(82.6 \%)$ \\
\hline Fever & $56(65.1 \%)$ \\
\hline Lack of appetite & $49(57.0 \%)$ \\
\hline Night Sweating & $48(55.8 \%)$ \\
\hline Malaise & $42(48.8 \%)$ \\
\hline Wight loss & $35(40.7 \%)$ \\
\hline Abdominal pain & $19(22.1 \%)$ \\
\hline Headache & $6(7.0 \%)$ \\
\hline Arthritis & $20(23.3 \%)$ \\
\hline Hepatosplenomegaly & $11(12.8 \%)$ \\
\hline Lymphadenopathy & $1(1.2 \%)$ \\
\hline \multicolumn{2}{|l|}{ Laboratory findings } \\
\hline Leukocytes, (/uL), median (IQR) & $8537(6317-9501)$ \\
\hline Absolute neutrophil count, (/uL), median (IQR) & 3505 (2505-5031) \\
\hline Hemoglobin, gr/dL, mean \pm SD & $13.4 \pm 1.5$ \\
\hline Thrombocyte, (/uL), mean \pm SD & $315621 \pm 84385$ \\
\hline C-reactive protein, median (IQR) (0-5 mg/dL) & $0.4(0.3-1.6)$ \\
\hline Erythrocyte sedimentation rate, mean $\pm \mathrm{SD}(0-20 \mathrm{~mm} / \mathrm{hr})$ & $13.3 \pm 11.4$ \\
\hline Maximum AST value, median (IQR) (5-34 U/L) & $27.5(18-45.7)$ \\
\hline Maximum ALT value, median (IQR) (0-55 U/L) & $23(16-38.7)$ \\
\hline Maximum GGT value, median (IQR) (12-64 U/L) & $21(14-28)$ \\
\hline Rose Bengal positivity & $68(79.0 \%)$ \\
\hline \multicolumn{2}{|l|}{ Brucellar tube agglutination test } \\
\hline$<1 / 160$ & $21(24.5 \%)$ \\
\hline $1 / 160$ & $16(18.6 \%)$ \\
\hline $1 / 320$ & $47(54.7 \%)$ \\
\hline $1 / 640$ & $0(0 \%)$ \\
\hline $1 / 1280$ & $1(1.2 \%)$ \\
\hline \multicolumn{2}{|l|}{ Brucellar Coomb's agglutination test } \\
\hline$<1 / 160$ & $9(10.5 \%)$ \\
\hline $1 / 160$ & $28(32.6 \%)$ \\
\hline $1 / 320$ & $15(17.4 \%)$ \\
\hline $1 / 640$ & $29(33.7 \%)$ \\
\hline $1 / 1280$ & $4(4.7 \%)$ \\
\hline Growth of brucella spp. on blood culture & $24(27.9 \%)$ \\
\hline Iron, mean $\pm S D,(65-175$ ug/dL) & $52.8 \pm 32.9$ \\
\hline Iron-binding capacity, mean \pm SD (120-370 ng/dL) & $261.1 \pm 55.5$ \\
\hline Ferritin, median (IQR) $(21.8-274.6 \mathrm{ng} / \mathrm{mL})$ & $77(38.5-223)$ \\
\hline Vitamin D level, mean \pm SD $(20.0-60.3 \mathrm{ng} / \mathrm{mL})$ & $19.3 \pm 8.6$ \\
\hline
\end{tabular}

Outcome after antibrucellar treatment 
7 th day, the cultures were incubated until the 21 st day. Subspecies of Brucella could not be identified in the hospital, and blood culture results were, therefore, given as Brucella spp. Children above 16 years old, with no blood culture, and diagnosed with other chronic diseases were excluded. The study was approved by the local Ethical Committee.

Patients' data including sociodemographic (age, gender, place of residence, etc.), clinical (duration of symptoms, presence of fever, malaise, night sweats, etc.), and laboratory (leukocyte, hemoglobin level, thrombocyte counts, etc.) characteristics were retrospectively included. Disease was classified as acute brucellosis when symptoms lasted for less than 8 weeks, subacute brucellosis when symptoms lasted between 2 and 12 months; and chronic brucellosis when symptoms lasted for more than 1 year. 6 Due to paucity of numbers, subacute and chronic cases were combined and defined as non-acute brucellosis.

All patients who used their medication properly were evaluated as cured in the absence of clinical signs and symptoms up to six months after the end of the antibrucellar treatment, as partial remission if existing signs and symptoms had decreased, and as relapse/ reinfection if they had signs and symptoms plus either growth of Brucella species in cultures and/or presence of positivity of serological parameters, at least six months after the end of the anti-brucellar treatment following an asymptomatic period.

The Kolmogorov-Smirnov test was used to determine the normality of the data. Numerical variables are expressed as mean \pm standard deviation and categorical variables as numbers (percentage). Median (inter-quartile range) values were used for non-normally distributed data. Student's $t$ test, the Mann-Whitney U-test, the Chisquare test and receiver operating characteristic (ROC) analyses were used as hypothesis tests. Children with Brucella spp. growing in blood culture were compared with children without Brucella spp. p-values $<0.05$ were considered statistically significant. ROC curves were used to examine the usefulness of significant variables in discriminating patients with positive blood culture from those with negative blood culture. Data were analysed on SPSS for Windows, version 17.0 software (SPSS, Chicago, IL).

\section{RESULTS}

The mean age of the children in the study was $10.9 \pm 3.9$ years, ranging between 1.2 and 16 years. Symptoms compatible with brucellosis were present for a median of 30 days. The demographic and clinical features, physical examination findings, and laboratory findings were shown in Table I. Seventy percent $(n=68)$ of the patients had positive Rose Bengal tests. Approximately onefourth of the patients had negative SAT and one tenth had negative Coombs test titers. Brucella spp. grew in blood culture of $24(27.9 \%)$ patients.

Univariate analyses showed that children with blood culture positivity had shorter symptom duration than those with negative blood cultures $(p=0.03$, Table II). Absence of a personal and household history of brucellosis were associated with blood culture positivity $(p=0.02$ and $p=0.04$, respectively). Lower hemoglobin, iron, and vitamin $D(p<0.001, p=0.006$, and $p=0.006$, respectively) and higher leukocyte, CRP, and ferritin $(p<0.001, p=0.001$, and $p<0.001$, respectively) levels were associated with isolation of Brucella spp. in blood culture. Children with positive blood cultures had higher SAT and Coombs agglutination test results than other children ( $p=0.001$ and $p<0.001$, respectively).

$\mathrm{ROC}$ analysis showed that based on the analysis of the parameters, leukocyte count, CRP, hemoglobin, iron, ferritin, and vitamin $D$ levels, and brucellar tube and Coombs agglutination tests could be used to predict blood culture positivity for brucellosis. Ferritin with a cutoff level of $122 \mathrm{ng} / \mathrm{mL}$ (Cl 95\% 0.86-0.97, p<0.001) and Brucella Coombs agglutination test at a cut-off level of $1 / 480(\mathrm{Cl} 95 \%$ 0.84-0.96, $p<0.001)$ were the most sensitive and specific predictors of bacteremia (Table III). Also this analysis showed that duration of symptoms was not significant for bacteremia in these children.

Table II: Risk factors related to blood culture positivity in children with brucellosis.

\begin{tabular}{|c|c|c|c|}
\hline Parameter & $\begin{array}{c}\text { Blood culture } \\
\text { Positive } \\
n=24\end{array}$ & $\begin{array}{c}\text { Blood culture } \\
\text { Negative } \\
n=62\end{array}$ & p-value \\
\hline Duration of symptoms, days, median (IQR) & $30(15-30)$ & $30(20-90)$ & 0.03 \\
\hline Presence of previous brucellosis (relapse/reinfection), $\mathrm{n}(\%)$ & $0(0 \%)$ & $11(100 \%)$ & 0.02 \\
\hline History of brucellosis in family members, $n(\%)$ & $16(38.1 \%)$ & $26(61.9 \%)$ & 0.04 \\
\hline Hemoglobin, gr/dL, median (IQR) & $12.5(11.3-12.9)$ & $13.9(13.1-14.9)$ & $<0.001$ \\
\hline Leukocytes, (/uL), median (IQR) & $11821(9027-14749)$ & $7401(5858-8914)$ & $<0.001$ \\
\hline CRP, (0-5 mg/dL), median (IQR) & $1.2(0.5-2.9)$ & $0.3(0.3-1.3)$ & 0.001 \\
\hline Iron, (65-175 ug/dL), median (IQR) & $32(23.2-42.7)$ & $48(30-90)$ & 0.006 \\
\hline Ferritin, (21.8-274.6 ng/mL), median (IQR) & $295(201-438)$ & $53(26.7-116)$ & $<0.001$ \\
\hline Vitamin D level, (15.7-60.3 ng/mL), median (IQR) & $14.9(10.8-17.5)$ & $19.6(14.6-25.9)$ & 0.006 \\
\hline Brucellar tube agg. test, median (IQR) & $320(320-320)$ & $160(20-320)$ & 0.001 \\
\hline Brucellar Coombs agg. test, median (IQR) & $640(640-640)$ & $160(160-320)$ & $<0.001$ \\
\hline
\end{tabular}

SD: Standard deviation; IQR: Interquartile range; CRP: C-reactive protein; agg: agglutination. 
Table III: Receiver operating characteristic analysis of the parameters as predictors of blood culture positivity for brucella.

\begin{tabular}{|c|c|c|c|c|c|c|}
\hline Variables & $\begin{array}{l}\text { Area under the curve } \\
(\%)\end{array}$ & $\begin{array}{c}\text { Asymptomatic } 95 \% \text { C } \\
(\%)\end{array}$ & Threshold value & Sensitivity & Specificity & $p$-value \\
\hline Duration of symptoms (days) & 37 & $24-49$ & 37.5 & 0.79 & 0.46 & 0.066 \\
\hline Hemoglobin level & 19 & $9-29$ & $12.9 \mathrm{gr} / \mathrm{dL}$ & 0.79 & 0.80 & $<0.001$ \\
\hline Iron level & 33 & $21-45$ & $38.5 \mathrm{ug} / \mathrm{dL}$ & 0.70 & 0.65 & 0.020 \\
\hline Brucella tube agg. test & 68 & $56-80$ & 240 & 0.79 & 0.50 & 0.010 \\
\hline C-reactive protein level & 70 & $59-82$ & $0.7 \mathrm{mg} / \mathrm{dL}$ & 0.66 & 0.64 & 0.003 \\
\hline Vitamin D level & 75 & $14-3$ & $17.5 \mathrm{ng} / \mathrm{mL}$ & 0.75 & 0.65 & $<0.001$ \\
\hline Leukocyte count & 86 & $77-95$ & 8970 /uL & 0.79 & 0.76 & $<0.001$ \\
\hline Brucella Coombs agg. test & 90 & $84-96$ & 480 & 0.91 & 0.81 & $<0.001$ \\
\hline Ferritin level & 92 & $86-97$ & $122 \mathrm{ng} / \mathrm{mL}$ & 0.91 & 0.78 & $<0.001$ \\
\hline
\end{tabular}

Cl: Confidence interval; agg: agglutination.

\section{DISCUSSION}

Brucellosis is still an important public health problem in Turkey, with a seroprevalance of $5 \%$ and a predilection to the eastern part of the country, including Erzurum. ${ }^{7}$ It is difficult to diagnose because the clinical manifestations are diverse and vague. Serological tests are easy to perform, economical, and time-saving. ${ }^{2}$ Nevertheless, definitive diagnosis requires the recovery of Brucella organisms from blood, bone marrow, or other tissue. 5 Obtaining a bone marrow culture is invasive and painful, despite its some advantages. Blood culture remains the method of choice in practical definitive diagnosis. In this study, Brucella spp. grew in blood culture from $27.9 \%$ of patients. Although Logan et al. 8 reported a high blood culture positivity level of $86 \%$, previous studies reported similar levels between $17.6 \%$ and $50.8 \%$ in children with brucellosis. 9,10 In general, growth of any micro-organism in blood culture depends upon previous use of antibiotics, culture methods, and the volume of the clinical specimen. ${ }^{11}$ The culture method and volume of the blood for cultures were standard for these study children.

The stage of the disease also affects the blood culture results. The time when blood is drawn is correlated with the detection of viable organisms in blood samples in brucellosis. Blood culture positivity levels as high as $80 \%$ to $90 \%$ in acute brucellosis decrease to $30 \%$ to $70 \%$ in chronic cases. ${ }^{12}$ In this study, although the possibility of isolating the bacterium in blood culture was higher during acute infection, duration of symptoms was not a predictive parameter for blood culture positivity at further analyses. Similarly, Giannakopoulos et al. reported no difference in duration of symptoms until admission between patients with positive and negative blood cultures. ${ }^{13}$ Also presence of fever at the time of blood drawn may interfere with blood culture results. Although fever has been associated with bacteremia in previous research, presence of fever was not related to the growth in blood culture in this study.6,14

Pathogens compete for iron with the host in order to colonize, proliferate, and cause disease, while the host benefits from the toxicity of iron to kill pathogens, in addition to its own metabolic pathways. Diversion of free iron to storage in the form of ferritin has been shown during infections caused by Salmonella and Mycobacterium. 15,16 Brucella spp. live inside the macrophages and require iron. They obtain this from the host in several ways, and excess intracellular iron is usually stored in the form of ferritin and/or bacterioferritin for release for cellular metabolism when required. 17 In general, as with CRP, elevation of ferritin levels occurs due to inflammatory response against infections. Arica et al. reported very high ferritin levels in pediatric patients with brucellosis. ${ }^{18}$ These then returned to normal ranges as the patients' symptoms decreased with treatment. Bayraktar et al. showed that serum iron levels decreased, while CRP and ferritin levels were significantly increased in patients with brucellosis. ${ }^{19}$ Similarly, in this study patients with positive blood culture had lower iron and higher ferritin levels than the other subjects. Ferritin with a cut-off level of $122 \mathrm{ng} / \mathrm{mL}$ was the most powerful predictor of bacteremia in children with brucellosis, with sensitivity of $91 \%$ and specificity of $78 \%$. Anemia is one of the most common laboratory findings in brucellosis. ${ }^{20}$ Anemia of chronic disease, characterised by low serum iron and increased iron retention within the mononuclear phagocyte system, is established by the host during infections. ${ }^{21}$ In this study, culture positive patients also had lower hemoglobin levels than culture negative patients, in agreement with Apa et al. ${ }^{14}$ Hemoglobin levels can also be used to predict blood culture positivity for brucellosis.

In this study, increased leukocyte and CRP levels were determined in patients with positive blood cultures, similarly to Apa et al. ${ }^{14}$ The ROC analyses also showed that CRP levels may be a promising parameter in predicting blood culture positivity in children with brucellosis. Differently, Kadanali et al. 6 observed no association between high CRP levels and bacteremia, while increased transaminases and leukopenia were correlated with blood culture positivity in brucellosis patients.

The brucellar tube and Coombs agglutination tests were significantly more positive in the bacteremic patients compared to the culture-negative group in this study. Unfortunately, the similarity between the O-antigenic side chain of LPS of Brucella and other organisms, such 
as Yersinia enterocolitica, Vibrio cholerae, Esherichia coli, and Francisella tularensis may result in false positivity. 2 In this study, the brucellar tube and Coombs agglutination tests emerged as powerful predictors of bacteremia. In contrast, the Rose Bengal test, which is used for screening because of its rapidity and easy applicability, was not associated with blood culture positivity. This test is highly sensitive, but its specificity varies between $75 \%$ and $91 \%$, and positive results are confirmed by the serum agglutination tests. ${ }^{22}$

Vitamin $D$ is an essential micronutrient for various body functions, such as bone metabolism and the immune system. Deficiency has been linked to various types of disorder, such as several types of cancer and diabetes mellitus. ${ }^{23}$ Vitamin $D$ deficiency inhibits macrophage functioning, such as chemotaxis, phagocytosis, and proinflammatory cytokine production and an association with infections has also been reported in children.24 Brucella cannot, therefore, be killed by inactivated macrophages. Kurtaran et al. showed that adult brucellosis patients had lower levels of vitamin $D$ and its receptor compared to a control group. 25 In this study, blood culture positivity was related to low vitamin $D$ levels. It also showed that vitamin D levels $<17.5 \mathrm{ng} / \mathrm{mL}$ could be used as a marker of blood culture positivity in children with brucellosis.

A positive family history and living in a rural area are risk factors for relapse in brucellosis. However, a history of brucellosis in family members was not correlated with growth in blood culture, similarly to the presence of relapse/reinfection type brucellosis. The mean age of the children in the present study was 10.9 years, similar to that in Giannakopoulos et al.13 They reported that patients with positive blood cultures were younger than those with negative blood cultures. Apa et al. reported similar findings. Nevertheless, no such association was observed in this study. ${ }^{14}$

This study has also some limitations. This is a retrospective study and statistically significant results were not achieved in some parameters, due to the small size of the study population. B. melitensis was almost the only pathogen found in previous studies.8,10 Subtype analysis could not be performed in the current study, which prevented analysis of more specific risk factors. However, to the best of authors' knowledge, this is the first trial to date to show a connection between vitamin $D$ deficiency and positive blood culture for Brucella.

\section{CONCLUSION}

Some biochemical and serological parameters, such as serum hemoglobin, iron, ferritin, vitamin D, C-reactive protein levels, Brucellar tube and Coombs agglutination tests, and leukocyte count are important parameters predicting blood culture positivity in pediatric brucellosis when molecular techniques are not feasible, such as in source-limited countries. Patient age, duration of symptoms, and presenting symptoms may not affect blood culture positivity. At the same time, blood culture positivity may not be associated with increased brucellosis-associated complications.

Disclosure: This manuscript was presented as an oral presentation in the $10^{\text {th }}$ Turkish National Pediatric Infectious Diseases Congress, April 5-9, 2017, Belek/ Antalya (S-10).

\section{REFERENCES}

1. Franco M, Mulder M, Gilman R, Smits HL. Human brucellosis. Lancet Infect Dis 2007; 7:775-86.

2. Christopher S, Umapathy BL, Ravikumar KL. Brucellosis: review on the recent trends in pathogenicity and laboratory diagnosis. J Lab Physicians 2010; 2:55-60.

3. Shemesh AA, Yagupsky P. Limitations of the standard agglutination test for detecting patients with Brucella melitensis bacteremia. Vector Borne Zoonotic Dis 2011; 11:1599-601.

4. Vrioni G, Pappas G, Priavali E, Gartzonika C, Levidiotou S. An eternal microbe: Brucella DNA load persists for years after clinical cure. Clin Infect Dis 2008; 46:131-6.

5. Young EJ. Brucella Species (Brucellosis). In: Long SS, Pickering LK, Prober CG (Eds). Principles and practice of pediatric Infectious diseases. ed. $7^{\text {th }}$. Churchill Livingstone, Pennsylvania; 2012: p.876-80.

6. Kadanali A, Ozden K, Altoparlak U, Erturk A, Parlak M. Bacteremic and nonbacteremic brucellosis: clinical and laboratory observations. Infection 2009; 37:67-9.

7. Public Health Specialists Association (HASUDER). Turkish Health Report 2014. Available at: http://halksagligiokulu.org/ anasayfa/components/com_booklibrary/ebooks/TSR2014T.PDF, (last accessed 20/03/2018).

8. Logan LK, Jacobs NM, McAuley JB, Weinstein RA, Anderson EJ. A multicenter retrospective study of childhood brucellosis in Chicago, Illinois from 1986 to 2008. Int J Infect Dis 2011; 15:e812-7.

9. Fruchtman Y, Segev RW, Golan AA, Dalem Y, Tailakh MA, Novak $\mathrm{V}$, et al. Epidemiological, diagnostic, clinical, and therapeutic aspects of brucella bacteremia in children in southern Israel: A 7-year retrospective study (2005-2011). Vector Borne Zoonotic Dis 2015; 15:195-201.

10. Tanir G, Tufekci SB, Tuygun N. Presentation, complications, and treatment outcome of brucellosis in Turkish children. Pediatr Int 2009; 51:114-9.

11. Aliskan $\mathrm{H}$. The value of culture and serological methods in the diagnosis of human brucellosis. Mikrobiyol Bul 2008; 42: 185-95.

12. Al Dahouk S, Nöckler K. Implications of laboratory diagnosis on brucellosis therapy. Expert Rev Anti Infect Ther 2011; 9: 833-45.

13. Giannakopoulos I, Nikolakopoulou NM, Eliopoulou M, Ellina A, Kolonitsiou F, Papanastasiou DA. Presentation of childhood brucellosis in western Greece. Jpn J Infect Dis 2006; 59:160-3.

14. Apa H, Devrim I, Memur S, Günay I, Gülfidan G, Celegen M, et al. Factors affecting Brucella spp. blood cultures positivity in children. Vector Borne Zoonotic Dis 2013; 13:176-80. 
15. Nairz M, Theurl I, Ludwiczek S, Theurl M, Mair SM, Fritsche G, et al. The coordinated regulation of iron homeostasis in murine macrophages limits the availability of iron for intracellular Salmonella typhimurium. Cell Microbiol 2007; 9:2126-40.

16. Silva-Gomes $S$, Bouton C, Silva T, Santambrogio P, Rodrigues $P$, Appelberg $\mathrm{R}$, et al. Mycobacterium avium infection induces $\mathrm{H}$-ferritin expression in mouse primary macrophages by activating Toll-like receptor 2. PLoS One 2013; 8:e82874.

17. Almirón MA, Ugalde RA. Iron homeostasis in Brucella abortus: The role of bacterioferritin. J Microbiol 2010; 48:668-73.

18. Arica V, Silfeler I, Arica S, Tutanç M, Motor VK, Inci M. Brucellosis with very high ferritin levels: Report of five cases. Hum Exp Toxicol 2012; 31:104-6.

19. Bayraktar M, Bayraktar N, Bayindir Y, Durmaz R. Serum C-reactive protein in patients with brucellosis, iron and ferritin levels in the diagnosis and follow-up value. Ankem J 2005; 19:61-3.

20. Buzgan T, Karahocagil MK, Irmak H, Baran Al, Karsen H, Evirgen $\mathrm{O}$, et al. Clinical manifestations and complications in
1,028 cases of brucellosis: A retrospective evaluation and review of the literature. Int J Infect Dis 2010; 14:e469-78.

21. Silva-Gomes S, Vale-Costa S, Appelberg R, Gomes MS. Iron in intracellular infection: To provide or to deprive? Front Cell Infect Microbiol 2013; 3:96.

22. Ruiz-Mesa JD, Sanchez-Gonzalez J, Reguera JM, Martín L, Lopez-Palmero S, Colmenero JD. Rose Bengal test: Diagnostic yield and use for the rapid diagnosis of human brucellosis in emergency departments in endemic areas. Clin Microbiol Infect 2005; 11:221-5.

23. Holick MF, Chen TC. Vitamin D deficiency: A worldwide problem with health consequences. Am J Clin Nutr 2008; 87:1080-6.

24. Cayir A, Turan MI, Ozkan O, Cayir Y, Kaya A, Davutoglu S, et al. Serum vitamin $D$ levels in children with recurrent otitis media. Eur Arch Otorhinolaryngol 2014; 271:689-93.

25. Kurtaran B, Akyildiz O, Candevir Ulu A, Inal SA, Komur S, Seydaoglu G, et al. The relationship between brucellosis and vitamin D. J Infect Dev Ctries 2016; 10:176-82.

.......... 